\title{
Introduction of the Novel Radiographic Line (L5-Kyphosis Apex Line) Intended to Evaluate Scheuermann's Disease and Postural Kyphosis Progression on Standard Lateral X-Rays
}

\author{
Tomer Bezalel $^{1,2}$, Eli Carmeli ${ }^{3}$, Leonid Kalichman ${ }^{2}$ \\ ${ }^{I}$ Maccabi Health Care Services, Posture Clinic, Maccabi Hashalom, Tel Aviv, Israel \\ ${ }^{2}$ Department of Physical Therapy, Recanati School for Community Health Professions, Faculty of Health Sciences at Ben-Gurion University of the Negev, \\ Beer-Sheva, Israel \\ ${ }^{3}$ Department of Physical Therapy, Faculty of Social Welfare and Health Sciences, Haifa University, Mt Carmel, Israel
}

Study Design: Cross-sectional and follow-up nested study.

Purpose: To assess the reliability of the L5-kyphosis apex line (L5-KAL) evaluation and determine the association between changes in L5-KAL, changes in radiological parameters, and changes in clinical symptoms.

Overview of Literature: Scheuermann's disease is the most common cause of hyperkyphosis of the thoracolumbar and thoracic spine during adolescence. Scheuermann's disease patients usually show compensatory hyperlordosis of the lumbar spine, which is usually flexible, and express an anterior translation of the lumbar section. The L5-KAL was developed on the basis of our clinical experience, displaying the horizontal distance between the thoracic and lumbar curves.

Methods: In the cross-sectional segment, 150 initial lateral X-rays of patients with Scheuermann's disease and postural kyphosis were analyzed, and 80 additional $X$-rays were analyzed in the follow-up segment. The data taken from the X-rays of the whole spinal column included the thoracic kyphosis angle, the C7 plumb line, and the L5-KAL. Clinical data included a numerical rating scale of self-perceived body image, pain, and a Scoliosis Research Society-22 questionnaire (SRS-22).

Results: Significant positive associations were observed in a cross-sectional study between the L5-KAL and thoracic kyphosis, lumbar lordosis, C7 line, and self-perceived body image. In the follow-up nested study, in a mixed analysis of variance, the main effect of time was significant for the L5-KAL, kyphotic deformity and SRS-22, which indicated a change in these parameters after treatment of Scheuermann's disease patients. Significant positive associations were observed between the changes in L5-KAL and the changes in thoracic kyphosis.

Conclusions: Our evidence suggests that the L5-KAL can serve as an indicator of the thoracic curve change in Scheuermann's disease and postural kyphosis patients and should be considered in clinical practice.

Keywords: Scheuermann's disease; X-rays; Kyphosis; Spine

Received Jun 28, 2019; Revised Jul 27, 2019; Accepted Jul 31, 2019

Corresponding author: Leonid Kalichman

Department of Physical Therapy, Recanati School for Community Health Professions, Faculty of Health Sciences, Ben-Gurion University of the Negev, P.O.B. 653, Beer Sheva, 84105, Israel

Tel: +972-52-2767050, Fax: +972-8-6477683, E-mail: kleonid@bgu.ac.il, kalichman@hotmail.com 


\section{Introduction}

Scheuermann's disease, the most common cause of hyperkyphosis of the thoracic and thoracolumbar spine, occurs during adolescence. After idiopathic scoliosis, it is the most common disorder in patients with a deformity of the spine $[1,2]$. The reported prevalence of Scheuermann's kyphosis ranges from $0.4 \%$ to $8 \%$ in the population [3-6]. The definition of Scheuermann's disease has been modified over the years; however, Sorenson's specific criteria of three or more adjacent apical vertebrae wedged at a minimum of $5^{\circ}$ each, with a thoracic kyphosis $>45^{\circ}$ (T3-T12), have been used predominately [3]. From a radiological perspective, Scheuermann's disease is characterized by vertebral body wedging, diminished anterior vertebral growth, vertebral endplate irregularity, narrowing of the intervertebral disk spaces, Schmorl's nodes, and premature disc degeneration $[4,7]$.

Aesthetic appearance/deformity of the back is the most frequent complaint of Scheuermann's disease patients and is typically the primary reason younger patients seek medical attention [8]. Pain, when present, is usually mild, precipitated by prolonged periods of sitting or exercise, and is typically located near the apex of the deformity. In general, the pain subsides with the cessation of growth. Occasionally, low back pain may be associated with spondylolisthesis, which is observed with increased incidence in Scheuermann's disease patients [9].

Clinically, Scheuermann's disease patients commonly display compensatory hyperlordosis of the lumbar spine. The lumbar deformity is usually flexible and finds expression in the anterior translation of the lumbar section ("swayback"), which is very common in Scheuermann's disease patients and can be dramatically influenced by the relative position of the thoracic and lumbar spine (transitional zone). In addition to a number of wedged vertebrae and the Cobb angle of the thoracic kyphosis, various other radiographical characteristics of Scheuermann's disease can be found in the literature; i.e., the C7 plumb line, which describes the radiological sagittal balance [5]. The sagittal balance assesses the association between the lower cervical spine and the sacral promontory but does not relate to the relative position of the thoracic and lumbar spine. The $\mathrm{C} 7$ plumb line is a vertical line drawn from the middle of the body of the $\mathrm{C} 7$ vertebral body. When the spine is balanced in the sagittal plane, the $\mathrm{C} 7$ plumb line should lie within $2 \mathrm{~cm}$ of the sacral promontory. However, patients with Scheuermann's disease tend to be negatively balanced compared with the general population, and therefore, the C7 plumb line lies behind the sacral promontory [10]. In some Scheuermann's disease patients, even with advanced pathology, the full compensation between thoracic kyphosis and lumbar and cervical lordosis can be observed albeit the $\mathrm{C} 7$ plumb line still being in the normal range. In these cases, an additional radiological method of spinal deformity evaluation should be considered.

On the basis of clinical observations in the posture clinic, we found numerous Scheuermann's disease patients with an unbalanced lumbar region compared with the thoracic block. These observations were also found on $\mathrm{X}$-rays. Since there have been no studies in the literature describing a radiological index sensitive to the relative position of the thoracic and lumbar spine (transitional zone) in Scheuermann's disease patients, we therefore developed a new radiological index called the L5-kyphosis apex line (L5-KAL) (Fig. 1A, B) that fulfills the aforementioned criterion and also acts as an indicator of thoracic curve changes (disease progression). In the posture clinic, we observed significant changes in the L5-KAL in patients with Scheuermann's disease as well as with postural ky-
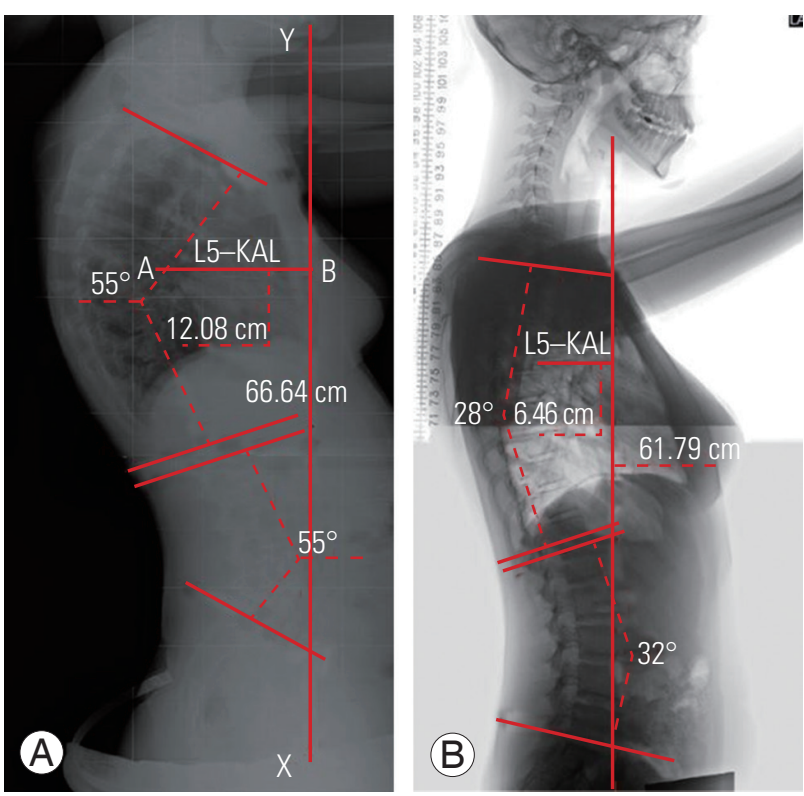

Fig. 1. (A) Initial evaluation. The thoracic kyphosis Cobb angle was $55^{\circ}$, and the lumbar lordosis Cobb angle was $55^{\circ}$. The $A B$ line (12.08 $\mathrm{cm}$ ) represents the new radiologic line (L5-KAL). (B) Follow-up evaluation (after Schroth therapy intervention). The thoracic kyphosis Cobb angle was $28^{\circ}$, and the lumbar lordosis Cobb angle was $32^{\circ}$. The $A B$ line $(6.46 \mathrm{~cm})$ represents the new radiologic line (L5-KAL). L5-KAL, L5kyphosis apex line. 
phosis, who reported improved clinical symptoms. In addition, their kyphotic Cobb angle was reduced.

Our hypothesis was that the L5-KAL is associated with the Cobb angle and the symptomatic representation of Scheuermann's disease and postural kyphosis patients can, therefore, be used as a tool to evaluate patients' kyphosis clinical and radiological progression.

The aims of the present study were as follows: (1) to assess the reliability of evaluation of the L5-KAL line; (2) to evaluate the association between the L5-KAL line and thoracic kyphosis curve (Cobb angle), sagittal balance (C7 plumb line), pain, and self-perceived body image (SPBI); and (3) to evaluate the association between L5-KAL changes, changes in the thoracic kyphosis curve (Cobb angle), and changes in the symptomatic representation of Scheuermann's disease and postural kyphosis (pain, SPBI, and Scoliosis Research Society-22 questionnaire [SRS22]).

\section{Materials and Methods}

\section{Study design}

The study comprised two components: (1) a cross-sectional study and (2) a follow-up nested study.

\section{Sample}

In the cross-sectional study, 150 lateral X-rays of males and females with Scheuermann's disease, who were diagnosed and treated in the Maccabi Hashalom Posture Clinic, were retrieved from the digital archives of the Maccabi Health Care Services, Tel Aviv, Israel. These subjects were diagnosed with Scheuermann's disease by a board-certified orthopedic surgeon, and they had been treated in the specialized posture clinic during the last 7 years (20092016). None had been surgically treated, and 21 had been treated with a brace. Maccabi Health Care Services is the second-largest health maintenance organization in Israel; therefore, this sample can be considered as representative of the general population of Scheuermann's disease patients. All X-rays were performed using a standard technique by specially trained technicians at the Department of Radiology, Maccabi Health Care Services Centers. The lateral view X-rays of the whole spinal column were taken in a standing position, with arms straight ahead in a $90^{\circ}$ shoulder flexion. Patients were instructed to stand natu- rally and place equal weight on both feet. In addition to the radiological parameters (the thoracic kyphosis angle, C7 line, and L5-KAL), SPBI, pain experienced during the last week, and the responses to the SRS-22 were analyzed from the patient's medical files and X-rays.

In the follow-up nested study, we used data from the randomized controlled trial performed at the Maccabi Hashalom Posture Clinic in Tel Aviv between 2013 and 2016. In this study, participants in both groups received a course of individual physical therapy sessions during 6-12 weeks, one appointment per week. One group received Schroth therapy exercises, and the second was treated by classic anti-gravitation exercises. No one received a bracing treatment during this study. We subsequently evaluated the L5-KAL, the thoracic Cobb angle, the responses to the SRS-22, pain experienced during the last week, SPBI, and kyphotic deformity measured by an inclinometer.

All patients were re-assessed after the intervention, and all of the parameters were measured again. The L5-KAL, the thoracic Cobb angle, the responses to the SRS-22, pain experienced during the last week, SPBI, and kyphotic deformity measured by an inclinometer were calculated pretreatment, after 6 months, and 1 year later. Eighty lateral $\mathrm{X}$-rays of 40 patients with Scheuermann's disease and postural kyphosis (two X-rays of each patient performed 6 to 12 months apart) were analyzed for this part of the study. Lateral X-rays were performed on all patients, before and after treatments.

\section{Imaging data collection}

The following data were collected from the lateral Xrays of the whole spinal column while standing: (1) The thoracic kyphosis angle (T3-T12) was measured with a modified Cobb method. The thoracic Cobb angle was measured from the T3 superior endplate to the T12 inferior endplate, indicating that the upper line was drawn at the superior endplate of $\mathrm{T} 3$ and the lower line at the inferior endplate of T12. (2) Sagittal balance (C7 plumb line) is the distance between the $\mathrm{C} 7$ vertebral body vertical axis and sacral promontory measured by drawing a vertical line from the middle of the body of the $\mathrm{C} 7$ vertebral body downwards and then calculating the distance between this line to the superior posterior (part of the sacral promontory). (3) Lumbar lordosis (L1-L5) was measured with a modified Cobb method. The lumbar Cobb angle was measured from the L1 superior endplate to the S1 supe- 
rior endplate. Lumbar lordosis was measured only in the cross-sectional study. (4) L5-KAL is the distance between the vertical line from the L5 anterior vertebral body to the anterior border of the thoracic kyphosis apex vertebral body, as previously mentioned.

\section{Reliability of the $\mathrm{X}$-ray readings}

Intra-rater reliability was assessed on the first $20 \mathrm{X}$-rays twice within a 2 -week interval by a physical therapist (T.B.), an expert in spinal deformity evaluation and treatment, with $>17$ years of experience in spinal X-ray evaluation. This analysis revealed the reliability of $0.99 \pm 8.42$ for the thoracic kyphosis angle, $0.99 \pm 3.64$ for the C7 line, and $0.99 \pm 3.06$ for the L5-KAL. These results demonstrate excellent reproducibility of the measurements. Twenty $\mathrm{X}$-rays were examined twice within a 2 -week interval time period.

\section{Clinical data collection}

SPBI: SPBI was evaluated using a numeric scale of $0-10$ (satisfied to very dissatisfied with appearance). A similar method of SPBI has been validated in other areas of medicine [11]. Pain: The amount of pain experienced the week prior was measured by the numeric pain rating scale (NPRS; $0=$ "no pain at all" and 10="the worst possible pain") [10]. Studies have shown that the NPRS scale is a valid and reliable tool for assessing pain in Scheuer- mann's disease patients $[6,12]$. Health-related quality of life: The SRS-22 is reliable, with internal consistency and reproducibility, and comparable with the 36-item ShortForm Health Survey (SF-36). The internal consistency of the SRS-22 (Cronbach $\alpha$ ) was 0.92 to 0.75 and that of the SF-36 was 0.91 to 0.36 . Reproducibility (intra-class correlation coefficient) of the SRS-22 was 0.96 to 0.85 and that of the SF-36 was 0.92 to 0.61 . Concurrent validity, determined by the Pearson correlation coefficients between the SRS-22 and SF-36 domains, was $\geq 0.70(p<0.0001)$ in 17 relevant comparisons [13].

\section{Ethical considerations}

The research was approved by the ethical (Helsinki) committee of Maccabi Healthcare Services (N. 0014-16-BBL) and by the IRB of Ben-Gurion University. For the followup part of the study, the parents of the patients, in the custodian position, signed an informed consent form according to the laws of medical ethics.

\section{Statistical analysis}

Statistical analysis was performed using IBM SPSS software ver. 21.0 for Windows (IBM Corp., Armonk, NY, USA). The mean and standard deviation for age, height, thoracic kyphosis, pain, body image, L5-KAL, SRS-22, and kyphotic deformity by inclinometer were calculated with descriptive statistics. We employed univariate analy-

Table 1. Demographic and baseline clinical characteristics of the cross-sectional and follow-up sample

\begin{tabular}{|c|c|c|}
\hline Characteristic & Value & No. of patients \\
\hline Age (yr) & $15.47 \pm 6.44$ & 200 \\
\hline Height (m) & $1.62 \pm 0.10$ & 176 \\
\hline Thoracic kyphosis $\left({ }^{\circ}\right)$ & $59.32 \pm 8.6$ & 200 \\
\hline Pain felt during the last week (numeric pain-rating scale) & $2.83 \pm 2.94$ & 177 \\
\hline Body image (numeric rating scale) & $4.62 \pm 3.6$ & 177 \\
\hline L5-kyphosis apex line (cm) & $11.79 \pm 2.69$ & 188 \\
\hline Scoliosis Research Society-22 questionnaire & $42.26 \pm 7.01$ & 50 \\
\hline Kyphotic deformity $\left.{ }^{\text {a) }}{ }^{\circ}\right)$ & $56.98 \pm 7.72$ & 50 \\
\hline \multicolumn{3}{|l|}{ Categorical variables } \\
\hline Risser sign & $4(2-4)$ & 197 \\
\hline Sex (males) & 58.5 & 200 \\
\hline
\end{tabular}

Values are presented as mean \pm standard deviation, median (25 percentile-75 percentile), or $\%$.

a) Measured by the digital inclinometer. 
ses to test the associations between the parameters in the cross-sectional section and between the changes in the parameters (delta values) in the follow-up nested section. Correlations between continuous variables were tested using Pearson's R; correlations between ordinal and continuous variables were tested with Spearman's Rho. In the follow-up section, the changes after treatment of the thoracic kyphosis, L5-KAL, SRS-22, and kyphotic deformity by inclinometer were measured using a mixed analysis of variance to test for the main effect of time. Because significant differences in sex, height, and age between the two groups were found, we introduced these variables as covariates in the mixed models to adjust for their effect on the outcome measures. The effect size was calculated using the partial eta squared measure.

\section{Results}

The total study sample included 190 participants (mean age, $15.47 \pm 6.64$ years) (Table 1); 150 were from the crosssectional study, and 40 were from the follow-up sample; $58.5 \%$ were males and $41.5 \%$ females. The mean L5-KAL was $11.79 \pm 2.69 \mathrm{~cm}$, and the mean thoracic kyphosis Cobb angle was $59.32^{\circ} \pm 8.6^{\circ}$.

Associations between the L5-KAL and kyphosis-related parameters measured in the cross-sectional section are shown in Table 2. Significant positive associations were observed between the L5-KAL and thoracic kyphosis $(r=0.445, p<0.001)$, lumbar lordosis $(r=0.523, p<0.001)$, C7 line $(r=0.722, p<0.001)$, and SPBI $(r=0.251, p=0.004)$.

The follow-up nested study revealed a significant main effect of time for the L5-KAL $(F[1]=4.29, p=0.04)$, kyphotic deformity measured by the inclinometer $(F[1]=42.87$, $p<0.01)$, and the SRS-22 $(F[1]=18.66, p<0.01)$, indicating that these measures were lower after treatment than those before treatment (Table 3).

Correlations between the L5-KAL change, the radiological parameter change, and the clinical symptomatic change are shown in Table 4. Significant positive associations were observed between the L5-KAL change, thoracic kyphosis Cobb angle change $(r=0.555, p<0.001)$, and the kyphotic deformity change measured by a digital inclinometer $(r=0.403, p=0.016)$.

Table 2. Correlations between the L5-kyphosis apex line and kyphosis-related parameters

\begin{tabular}{lcc} 
& & Correlations \\
Variable & $r$ & $p$-value \\
\cline { 2 - 3 } Thoracic kyphosis $\left({ }^{\circ}\right)$ & 0.445 & $<0.001$ \\
Lumbar lordosis $\left({ }^{\circ}\right)$ & 0.523 & $<0.001$ \\
C7 line (cm) & 0.722 & $<0.001$ \\
Pain (numeric pain-rating scale) & -0.014 & 0.875 \\
Self-perceived body image (numeric rating scale) & 0.251 & 0.004 \\
\hline
\end{tabular}

Statistically significant differences $(p<0.05)$ marked in bold. Correlations between continuous variables were tested using the Pearson's R. Correlations between ordinal and continuous variables were tested using the Spearman's Rho.

Table 3. Change of outcome measures due to treatment in a nested follow-up section

\begin{tabular}{|c|c|c|c|c|c|}
\hline \multirow{2}{*}{ Variable } & \multirow{2}{*}{ Baseline } & \multirow{2}{*}{ End of treatment } & \multicolumn{2}{|c|}{ Main effect of time } & \multirow{2}{*}{ Partial $\eta^{2}$} \\
\hline & & & $F$ & $p$-value & \\
\hline Thoracic kyphosis ${ }^{a)}\left({ }^{\circ}\right)$ & $58.13 \pm 8.01$ & $52.69 \pm 8.67$ & $F(1)=3.31$ & 0.07 & 0.07 \\
\hline L5-kyphosis apex line ${ }^{\text {a) }}(\mathrm{cm})$ & $11.48 \pm 2.66$ & $10.75 \pm 2.97$ & $F(1)=4.29$ & 0.04 & 0.11 \\
\hline Kyphotic deformity ${ }^{\mathrm{b})}\left({ }^{\circ}\right)$ & $56.83 \pm 7.75$ & $49.87 \pm 6.92$ & $F(2)=42.87$ & $<0.01$ & 0.48 \\
\hline Scoliosis Research Society-22 questionnaire ${ }^{\text {b) }}$ & $42.26 \pm 7.02$ & $33.18 \pm 10.88$ & $F(2)=18.66$ & $<0.01$ & 0.27 \\
\hline
\end{tabular}

Values are presented as mean \pm standard deviation, unless otherwise stated. Statistically significant differences $(p<0.05)$ marked in bold. ANOVA, analysis of variance.

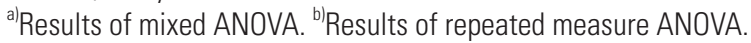


Table 4. Correlations between the changes in the L5-kyphosis apex line (cm) and changes in radiological parameters and clinical symptoms

\begin{tabular}{lcr} 
& & Correlations \\
Variable & $r$ & $p$-value \\
\cline { 2 - 3 } Scoliosis Research Society-22 questionnaire change & 0.047 & 0.784 \\
Thoracic kyphosis change $\left({ }^{\circ}\right)$ & 0.555 & $<0.001$ \\
Kyphotic deformity change $\left({ }^{\circ}\right)$ & 0.403 & 0.016 \\
Pain change (numeric pain-rating scale) & -0.057 & 0.743 \\
\hline Self-perceived body image change (numeric rating scale) & 0.039 & 0.823 \\
\hline
\end{tabular}

Statistically significant differences $(p<0.05)$ marked in bold. Correlations between continuous variables were tested using the Pearson's R. Correlations between ordinal and continuous variables were tested using the Spearman's Rho.

\section{Discussion}

The influence of sagittal alignment on clinical presentation (pain and deformity) and treatment of spinal deformities is important to achieve an optimum outcome of the treatment. The sagittal deformity can be assessed radiographically and clinically [14]. The radiographic assessment has a significant advantage over previously existing methods as it provides an accurate and objective evaluation of the deformity, thereby indicating the extent of correction required to achieve a normal balance. Several studies have investigated sagittal spinopelvis alignment as well as global spinal balance. Mac-Thiong et al. [15] explored the sagittal sacropelvic anatomy and regional sagittal spinal alignment in the pediatric population. In a subsequent study, Mac-Thiong et al. [16] reported on global spinal balance in asymptomatic adults and its influence on age, gender, and sacropelvic morphology using different parameters.

The L5-KAL, a new simple and reliable method of evaluating the relative position between the thoracic and lumbar spine, entails drawing a vertical line parallel to the L5 anterior vertebral body (demonstrated in Fig. $1 \mathrm{~A}$ as the $\mathrm{XY}$ line). The distance between this line and the anterior border of the thoracic kyphosis apex vertebral body (demonstrated in Fig. 1A as the AB line) is measured, displaying the horizontal distance between these two curves. The line accurately determines the anterior translation of the lumbar section ("swayback") caused by the compensatory hyperlordosis of the lumbar spine. The intra-rater reliability of the L5-KAL was excellent with high reproducibility of the results. Therefore, we believe that this line could be a useful tool for radiographically assessing patients with
Scheuermann's disease and postural kyphosis, on the side of other traditional measures of sagittal deformity.

The L5-KAL was able to detect the change in the relative position between the thoracic and lumbar spine in the follow-up study. In addition, significant associations were exhibited between the changes in the L5-KAL, changes in the Cobb angle of thoracic kyphosis, and the changes in a kyphotic deformity measured by an inclinometer and the clinical parameters. Therefore, L5-KAL may be used in orthopedic and physical therapy practice to monitor the change in thoracic curve changes in Scheuermann's disease and postural kyphosis patients. In addition, we believe that L5-KAL can be used for monitoring the thoracic curve changes in patients with age-related or osteoporotic kyphosis, but this should be assessed in future studies.

In some cases, i.e., when there is full compensation between the thoracic and lumbar curves, the conventional C7 plumb line is observed in the normal range. In such cases, the L5-KAL is much more advantageous than the C7 plumb line. In our clinical experience, and in the present study, we found that the change in L5-KAL is strongly associated with the changes in the SPBI in patients with Scheuermann's disease and postural kyphosis.

\section{Conclusions}

The L5-KAL is a new radiological line based on our clinical experience and on biomechanical principles. Our evidence suggests that the L5-KAL can serve as an indicator of thoracic curve changes and SPBI changes in patients with Scheuermann's disease and postural kyphosis and therefore can be applied in clinical practice. 


\section{Conflict of Interest}

No potential conflict of interest relevant to this article was reported.

\section{Author Contributions}

Each author is responsible for the content and accuracy of the entire manuscript. Tomer Bezalel: substantial contributions to the conception and design of the work; the acquisition, analysis, interpretation of data for the work; drafting the work; final approval of the version to be published. Eli Carmeli: substantial contributions to the conception of the work; revising the work critically for important intellectual content; final approval of the version to be published. Leonid Kalichman: substantial contributions to the conception and design of the work; revising the work critically for important intellectual content; final approval of the version to be published.

\section{References}

1. Holt RT, Dopf CA, Isaza JE. Adult kyphosis. In: Frymoyer JW, editor. The adult spine: principles and practice. 2nd ed. Philadelphia (PA): LippincottRaven; 1997. p. 1537-78.

2. Graat HC, van Rhijn LW, Schrander-Stumpel CT, van Ooij A. Classical Scheuermann disease in male monozygotic twins: further support for the genetic etiology hypothesis. Spine (Phila Pa 1976) 2002;27:E485-7.

3. Sorensen KH. Scheuermann's juvenile kyphosis: clinical appearances, radiography, aetiology, and prognosis. Copenhagen: Munksgaard; 1964.

4. Wenger DR, Frick SL. Scheuermann kyphosis. Spine (Phila Pa 1976) 1999;24:2630-9.

5. Lowe TG. Scheuermann's disease. Orthop Clin North Am 1999;30:475-87.

6. Ristolainen L, Kettunen JA, Heliovaara M, Kujala UM, Heinonen A, Schlenzka D. Untreated Scheuer- mann's disease: a 37-year follow-up study. Eur Spine J 2012;21:819-24.

7. Murray PM, Weinstein SL, Spratt KF. The natural history and long-term follow-up of Scheuermann kyphosis. J Bone Joint Surg Am 1993;75:236-48.

8. Bradford DS, Moe JH, Montalvo FJ, Winter RB. Scheuermann's kyphosis and roundback deformity: results of Milwaukee brace treatment. J Bone Joint Surg Am 1974;56:740-58.

9. Ogilvie JW, Sherman J. Spondylolysis in Scheuermann's disease. Spine (Phila Pa 1976) 1987;12:251-3.

10. Lowe TG, Kasten MD. An analysis of sagittal curves and balance after Cotrel-Dubousset instrumentation for kyphosis secondary to Scheuermann's disease: a review of 32 patients. Spine (Phila Pa 1976) 1994;19:1680-5.

11. Larsson P, John MT, Nilner K, List T. Reliability and validity of the Orofacial Esthetic Scale in prosthodontic patients. Int J Prosthodont 2010;23:257-62.

12. Weiss HR, Dieckmann J, Gerner HJ. Effect of intensive rehabilitation on pain in patients with Scheuermann's disease. Stud Health Technol Inform 2002;88:254-7.

13. Asher M, Min Lai S, Burton D, Manna B. The reliability and concurrent validity of the scoliosis research society-22 patient questionnaire for idiopathic scoliosis. Spine (Phila Pa 1976) 2003;28:63-9.

14. Roussouly P, Nnadi C. Sagittal plane deformity: an overview of interpretation and management. Eur Spine J 2010;19:1824-36.

15. Mac-Thiong JM, Berthonnaud E, Dimar JR 2nd, Betz RR, Labelle H. Sagittal alignment of the spine and pelvis during growth. Spine (Phila Pa 1976) 2004;29:1642-7.

16. Mac-Thiong JM, Roussouly P, Berthonnaud E, Guigui P. Sagittal parameters of global spinal balance: normative values from a prospective cohort of seven hundred nine Caucasian asymptomatic adults. Spine (Phila Pa 1976) 2010;35:E1193-8. 